\title{
Efectos del conflicto parental postdivorcio en la adaptación y bienestar de los hijos.
}

\section{Consequences of the parental conflict after the divorce on the adaptation and well-being of children.}

Fecha de recepción: 02-10-2017

Fecha de aceptación: 27-11-2017

\author{
Alicia Sanchis Castelló \\ Licenciada en Psicología. Master Universitario en Psicología \\ Jurídica. Preven3 \\ Cristina Robredo Torres \\ Servicio de Psiquiatría Hospital Casa de Salud, Valencia. \\ Raquel Llop Pérez \\ Centro Forenpsic, Valencia \\ Enrique J. Carbonell Vayá
}

Instituto Universitario en Criminología y Ciencias Penales. Universitat de València

\section{resumen/abstract:}

El debate acerca de la idoneidad de las separaciones y los nuevos matrimonios respecto al bienestar de los hijos sigue siendo un tema presente en la psicología. En esta investigación hemos querido observar la relación entre diversos factores considerados de riesgo, como la relación afectiva paterno-filial o el conflicto entre progenitores; y la adaptación de los menores a su entorno o su satisfacción familiar, así como analizar si se diferencian de la población general. Los resultados obtenidos muestran que la implicación de los menores en el conflicto parental y la discrepancia educativa entre ambos progenitores son los factores de mayor peso en la explicación de la insatisfacción familiar. Por otra parte, la insatisfacción familiar está directamente relacionada con la inadaptación personal, escolar y social de los menores evaluadas a través del TAMAl, aunque no se observan diferencias estadísticamente significas con la población de referencia.

The types of families are in flux nowadays due to the increasing number of blended families or single parents. Sometimes, this evolution raises a debate about the appropriateness of separations and remarriages for the well-being of the children. In this research, we've wanted therefore to observe the connection among several risk factors, such as the parent-child emotional relationship or a conflict between parents, the children's adaptation to their environment and their family satisfaction, as well as the differences with other children. The results show that the children's involvement in parental conflict and educational discrepancy between their parents are the factors that have the most considerable weight when it comes to explain the family dissatisfaction. On the other hand, family dissatisfaction is directly related to personal, social and academic maladjustment, although these results aren't higher than other children's results.

\section{palabras clave/keywords:}

Insatisfacción familiar, consecuencias, adaptación psicológica.

Familiar dissatisfaction, psychological adaptation, consequences. 


\section{Introducción}

Desde el ámbito de la psicología, conocer y entender lo mejor posible las causas y consecuencias de las disoluciones conyugales es cada vez más necesario, con el objetivo de mitigar al máximo los perjuicios que pueda suponer para cualquiera de los miembros de la familia, pero sobre todo para los hijos menores.

Al inicio de las investigaciones en este campo, el objeto central era la relación entre la ruptura de los cónyuges y la repercusión emocional en los hijos en comparación con familias intactas. En cambio, desde hace ya unos años se ha visto que es necesario tener en cuenta variables más concretas como las relaciones paterno-filiales previas, el nivel de conflicto entre progenitores, la percepción que tengan los menores de dicho conflicto, características individuales de los hijos, cambios en los recursos económicos familiares, estilo educativo, el tiempo que pasan los menores con cada progenitor, etc. (Catalán, 2015; Kelly y Emery, 2003; Fariña, Arce, Novo y Seijo, 2014). Amato (2014) propone que el malestar psicológico de los hijos puede ser consecuencia en parte de la separación como evento traumático y también resultado de factores de riesgo previos de los progenitores como por ejemplo rasgos de personalidad problemáticos o peores habilidades sociales.

Algunos de los estudios que comparan familias monoparentales o reconstituidas con familias intactas, indican que el divorcio supone con frecuencia un perjuicio en el estado psicológico de los menores (Fariña et al., 2014; Fariña, Martinón, Souto y García, 2014) que puede manifestarse a través de una mayor solicitud de atención psicológica y psiquiátrica (Amato, 2014; Seijo, Novo, Carracedo y Fariña, 2010), peor rendimiento académico (Catalán 2015; Novo, García y Carracedo, 2014), en forma de regresión o adquiriendo comportamientos más propios de adultos (Catalán, 2015). Además, Boone Holladay (2016) refiere que las consecuencias psicológicas para los menores son, con frecuencia, graves y duraderas.

En cambio, otras investigaciones señalan que el divorcio de los progenitores no siempre es un evento traumático o desagradable, sino que en ocasiones puede tener un efecto positivo sobre los hijos (Fariña, Seijo, Arce y Vázquez, 2017; Haimi y Lerner, 2016). En todo caso, en una investigación realizada por Morgado y González (2001) encontraron que unos cuatro años después de la ruptura de los progenitores, los menores se encontraban dentro de la media en competencia escolar, social, problemas de comportamiento y autoestima, diferenciándose de forma muy ligera de los hijos provenientes de familias intactas.

Investigaciones que tratan de hilar un poco más fino, hablan de que el hecho de que los padres rompan su relación no es el factor más relevante a la hora de explicar el bajo estado de ánimo y el desajuste de los hijos, sino que es la relación conflictiva entre los progenitores después de la separación la que ejerce una influencia directa en su bienestar (Tejedor, 2012) produciendo ansiedad, estrés, síntomas depresivos y/o problemas de inadaptación (Jiménez y Cano, 2014; Pons-Salvador, 2007; Pons-Salvador y Del Barrio, 1995). Además, se ha observado que el bienestar de los menores está más garantizado en una familia reconstituida en la que no hay conflicto que en una intacta con altos niveles de conflictividad (Catalán, 2015). 
Si los niños son testigos de frecuentes disputas entre sus padres, escuchan críticas de uno respecto al otro y, sobre todo, si se sienten parte de ese conflicto, es probable que presenten mayores niveles de ansiedad, miedo al abandono, problemas de conducta y depresión (Arch, 2010; Pons-Salvador, 2007).

Por supuesto, la ruptura produce confusión y malestar igual que cualquier otro evento vital estresante pero el proceso adaptativo puede ser satisfactorio si los recursos de la familia son los adecuados. Como prueba de ello, algunos estudios informan de que las parejas que se separan de forma saludable y proactiva, mantienen la relación con sus hijos prácticamente intacta (Arch, 2010, Fariña et al., 2017; Ríos-Sarrió, 2009). Otros factores protectores que han demostrado su efecto para paliar el desajuste postdivorcio son el estilo educativo asertivo, equilibrando la firmeza con el apoyo emocional (Catalán, 2015) y la adaptación psicológica a la separación del progenitor custodio (Kelly et al., 2003).

Otro factor importante en el proceso de adaptación de los niños es la relación previa a la separación que existía entre ellos y sus progenitores. Di Domenico (2006) realiza una pequeña revisión y encuentra que, si antes de la separación existe un vínculo de apego seguro entre padres e hijos, el niño no tiene miedo al abandono ni percibe que su relación con sus progenitores pueda deteriorarse, por ello lo más probable es que presente una regulación emocional adecuada, seguridad y confianza en sus relaciones afectivas y poca confusión sobre el futuro.

La edad que tengan los menores cuando se produzca la ruptura mediará en gran parte en su ajuste personal debido a su desarrollo cognitivo, que determinará en qué medida será capaz de entender la situación, y de su dependencia física y emocional, que harán más o menos fácil la separación temporal de uno de los progenitores en caso de ser necesaria (PonsSalvador, 2007). Por poner un ejemplo, se ha visto que los niños más pequeños tienden a autoinculparse y a presentar problemas conductuales como desobediencia o hiperactividad y los menores más cercanos a la adolescencia o adolescentes presentan más habitualmente problemas interpersonales y de adaptación social (Cantón, Cortés y Justicia, 2002).

Otro aspecto que ha preocupado a los expertos es la relación entre la modalidad de custodia y la adaptación de los hijos a la nueva situación familiar. Existen resultados indicativos de una mejor adaptación familiar, emocional, escolar y conductual en menores de parejas separadas cuyo régimen de convivencia posterior es compartido, comparado con menores en régimen de custodia monoparental (Bauserman, 2002; Fariña et al., 2017). Además, Nielsen (2014) realiza una revisión de 40 estudios sobre la idoneidad de cada régimen de custodia y concluye que la custodia compartida está relacionada también con mejor salud física y mejores relaciones familiares, incluso cuando el nivel de conflicto entre los padres era alto.

Sobre este particular, una revisión realizada por Catalán (2015) evidencia que la modalidad de custodia que mejor mantiene la estabilidad familiar es la compartida, pero siempre que los progenitores estén dispuestos a coordinarse, cooperar y colaborar, ya que los padres deben ponerse de acuerdo en mayor medida a la hora de tomar casi cualquier decisión sobre los hijos porque éstos pasan la misma cantidad de tiempo con cada uno. 
Al mismo tiempo, otros estudios muestran, por una parte, que en familias que han sufrido una separación saludable y no existe un conflicto entre los progenitores, las diferencias de comportamiento entre los hijos bajo custodia compartida y los hijos con custodia exclusiva son insignificantes. Y por otra parte, cuando la custodia es exclusiva y las visitas del progenitor no custodio son regulares, la adaptación, confianza y autoestima de los hijos también presentan niveles altos (Catalán, 2011).

Otros autores han encontrado además que los problemas emocionales y comportamentales de los hijos que conviven únicamente con uno de sus progenitores están mediados en su mayoría por los problemas de comunicación, supervisión y de demostración de afecto que pueda haber entre ellos (Cantón et al., 2002).

Esto podría indicar que la modalidad de custodia no ejerce un efecto directo sobre el bienestar de los hijos, sino que el estilo educativo, el estado emocional o la frecuencia de visitas, entre otras, podrían ser variables mediadoras de dicho efecto.

Como acabamos de exponer de forma resumida, existe una gran variedad de factores implicados en la separación y el divorcio matrimonial que podrían afectar de forma muy diferente al bienestar tanto de los hijos como de los padres. Por ello, uno de los objetivos de esta investigación es conocer si existen diferencias en cuanto a adaptación y bienestar psicológico de menores inmersos en procesos de separación conyugal en comparación con otros menores. Y, en su caso, discriminar cuales son las variables implicadas que se relacionan más directamente con estas diferencias.

\section{Método}

\section{Participantes}

En una primera fase de investigación se utilizó una muestra compuesta por un total de 177 sujetos de entre 8 y 17 años. El 32,2\% de la muestra lo componen chicas y el 67,8\% chicos. De ellos, 95 menores forman parte del grupo control o muestra comunitaria (evaluados en el contexto escolar de un centro concertado de la ciudad de Valencia) y 82 conforman el grupo experimental o muestra específica (evaluados en el contexto profesional privado de la provincia de Valencia). Este segundo grupo se divide, a su vez, en tres subgrupos: grupo clínico (menores evaluados por demanda terapéutica), grupo pericial familia (evaluados en el contexto judicial civil de familia) y grupo pericial otros (evaluados en el contexto pericial por otros motivos, en su mayoría por haber experimentado situaciones traumáticas).

En una segunda fase de la investigación, centrando la atención en el grupo de menores cuyos padres están separados o divorciados, se obtiene una muestra compuesta por 61 menores de entre 8 y 11 años, que denominaremos grupo pericial civil para diferenciarlo del anterior. El 47,54\% son chicas y el 52,46\% son chicos, evaluados en el contexto profesional privado de la provincia de Valencia. En esta muestra el 85,20\% de los menores parten de una custodia exclusiva materna previa, con niveles equilibrados o medios de contacto con el otro progenitor (23\% y $23 \%$ respectivamente) y contacto bajo o muy bajo en el $45,90 \%$ de los casos. No se registró ningún caso de abandono de la investigación a lo largo de todo el proceso. En todos los casos se solicitó y obtuvo el consentimiento para la evaluación ateniéndose a la patria potestad. 


\section{Procedimiento}

En la primera fase el principal objetivo es observar si existen diferencias en cuanto a adaptación y bienestar de los menores en todas sus áreas vitales (familiar, social, escolar y personal) en función del contexto en el que son evaluados (clínico, pericial civil de familia, pericial penal y escolar). Es decir, estudiar si los menores atendidos por una demanda terapéutica o pericial, y por tanto en situación de vulnerabilidad por diferentes motivos, (variables independientes) se diferencian en bienestar y adaptación (variables dependientes) de su población general de referencia. También se analizan las posibles diferencias que se puedan encontrar entre los subgrupos de la muestra específica, así como la relevancia del conflicto familiar y la implicación de los menores en él.

En la segunda fase de la investigación nos centramos en analizar el grupo de menores que están viviendo o han vivido la separación de sus progenitores, observando también si existen diferencias particulares en comparación con la población general. Concretamente, se pretende analizar si factores como el conflicto parental, la implicación de los hijos en él, el tiempo transcurrido desde la ruptura o el régimen de convivencia previo (variables independientes) ejercen una influencia en el bienestar psicológico de los menores evaluado a través de su satisfacción familiar y su adaptación en diferentes ámbitos (variables dependientes).

El primer paso fue recoger y categorizar la siguiente información de los expedientes de cada sujeto por parte de los profesionales que atendieron cada caso: datos demográficos y de identificación de los sujetos y sus familias, contexto de evaluación, nivel de conflicto entre los progenitores (ausencia o nivel bajo, nivel medio y nivel alto), nivel de implicación de los hijos en el conflicto parental (ausente o leve, media y alta), modalidad de guarda y custodia previa al procedimiento judicial y nivel de contacto con el progenitor no custodio (equilibrado, medio, bajo y nulo/PEF).

Para evaluar la relación paterno-filial desde la perspectiva de los hijos así como las posibles consecuencias que la situación familiar puede haber provocado en el estado y desarrollo de éstos se analizaron las siguientes variables del Test Autoevaluativo Multifactorial de Adaptación Infantil, TAMAI (Hernández, 2002): Inadaptación personal, Inadaptación escolar, Inadaptación social, Insatisfacción familiar, Insatisfacción con los hermanos, Educación adecuada del padre, Educación adecuada de la madre y Discrepancia educativa.

Para la primera fase de la evaluación se utilizaron los 3 niveles del cuestionario TAMAI (nivel I para menores de 8 a 11 años, nivel II de 12 a 14 años y nivel III de 15 a 18 años). Se analizaron las puntuaciones directas de todos ellos para evitar las diferencias de cálculo en los baremos de cada nivel. En la segunda fase solamente fue necesario el nivel I. El TAMAI tiene una fiabilidad global de 0.87 medida a partir del coeficiente Alfa de Cronbach.

\section{Análisis}

Para realizar los análisis correspondientes se ha utilizado el paquete estadístico SPSS 19.0. Con este programa se han realizado los análisis descriptivos, correlacionales (correlación de Pearson) y comparativos (ANOVA de un factor, T de Student, pruebas de contraste a posteriori, etc.) correspondientes en cada ocasión, tal y como se detalla en el apartado de resultados. 


\section{Resultados}

\section{Primera fase}

Respecto a los resultados de la primera investigación, en la tabla 1, se representan las medias, desviaciones típicas, F y significación del Anova, sobre la variable independiente Población de pertenencia en relación a las variables dependientes generales, es decir las puntuaciones de factores globalizantes de TAMAI. Se observa la clara pertenencia a un grupo más problemático de la muestra denominada específica ya que se observan niveles más altos de inadaptación general, escolar y social estadísticamente significativos. En cambio, estas diferencias no aparecen en la variable de inadaptación personal.

\section{Tabla 1}

Anova. Población general/específica en

Inadaptación

\begin{tabular}{|c|c|c|c|c|c|c|c|}
\hline & & $\mathrm{N}$ & Media & $\begin{array}{l}\text { Desviación } \\
\text { típica }\end{array}$ & $\begin{array}{l}\text { Error } \\
\text { típico }\end{array}$ & $\mathrm{F}$ & Sig. \\
\hline \multirow{3}{*}{$\begin{array}{l}\text { Inadaptación } \\
\text { General }\end{array}$} & Población General & 95 & 24.22 & 12.688 & 1.302 & \multirow{3}{*}{8.200} & \multirow{3}{*}{.005} \\
\hline & Población Específica & 82 & 30.07 & 14.501 & 1.601 & & \\
\hline & Total & 177 & 26.93 & 13.832 & 1.040 & & \\
\hline \multirow{3}{*}{$\begin{array}{l}\text { Inadaptación } \\
\text { Personal }\end{array}$} & Población General & 95 & 9.27 & 5.758 & .591 & \multirow{3}{*}{.056} & \multirow{3}{*}{.813} \\
\hline & Población Específica & 82 & 9.49 & 6.248 & .690 & & \\
\hline & Total & 177 & 9.37 & 5.974 & .449 & & \\
\hline \multirow{3}{*}{$\begin{array}{l}\text { Inadaptación } \\
\text { Escolar }\end{array}$} & Población General & 95 & 6.45 & 5.214 & .535 & \multirow{3}{*}{20.328} & \multirow{3}{*}{.000} \\
\hline & Población Específica & 82 & 10.48 & 6.645 & .734 & & \\
\hline & Total & 177 & 8.32 & 6.236 & .469 & & \\
\hline \multirow{3}{*}{$\begin{array}{l}\text { Inadaptación } \\
\text { Social }\end{array}$} & Población General & 95 & 8.49 & 4.290 & .440 & \multirow{3}{*}{5.571} & \multirow{3}{*}{.019} \\
\hline & Población Específica & 82 & 10.11 & 4.813 & .531 & & \\
\hline & Total & 177 & 9.24 & 4.598 & .346 & & \\
\hline
\end{tabular}

En la tabla 2 se representan los mismos análisis respecto a la valoración del entorno familiar. Podemos observar que en la muestra específica existen mayores dificultades y peor valoración del entorno familiar que en la población general (significativamente mayor insatisfacción familiar y discrepancia educativa; y peor valoración de ambos progenitores: educación adecuada de padre y madre significativamente más bajas). 


\section{Tabla 2}

Anova. Población general/específica en variables de ambiente familiar

\begin{tabular}{llcccccc}
\hline & $\mathrm{N}$ & Media & $\begin{array}{c}\text { Desviación } \\
\text { típica }\end{array}$ & $\begin{array}{c}\text { Error } \\
\text { típico }\end{array}$ & $\mathrm{F}$ & Sig. \\
\hline $\begin{array}{llcccc}\text { Insatis- } \\
\text { facción }\end{array}$ & Población General & 95 & .73 & 1.134 & .116 & & \\
familiar & Población Específica & 82 & 2.05 & 1.662 & .184 & 39.079 & .000 \\
& Total & 177 & 1.34 & 1.548 & .116 & & \\
$\begin{array}{l}\text { Insatisfac- } \\
\text { ción con }\end{array}$ & Población General & 95 & 1.14 & 1.264 & .137 & & \\
hermanos & Población Específica & 82 & 1.46 & 1.450 & .176 & 2.053 & .154 \\
& Total & 177 & 1.28 & 1.355 & .110 & & \\
Educación & Población General & 95 & 9.13 & 3.580 & .367 & & \\
adecuada & Población Específica & 82 & 5.56 & 6.400 & .707 & 21.648 & .000 \\
padre & 177 & 7.47 & 5.374 & .404 & & \\
Educación & Poblal & 95 & 10.00 & 2.666 & .274 & & \\
adecuada & Población General Específica & 82 & 8.22 & 4.969 & .549 & 9.151 & .003 \\
madre & Total & 177 & 9.18 & 3.994 & .300 & & \\
$\begin{array}{l}\text { Discrepan- } \\
\text { cia educa- }\end{array}$ & Población General & 95 & 2.47 & 2.828 & .290 & & \\
tiva & Población Específica & 82 & 7.48 & 10.254 & 1.132 & 20.791 & .000 \\
& Total & 177 & 4.79 & 7.676 & .577 & & \\
\hline
\end{tabular}

En las variables dependientes genéricas de inadaptación los subgrupos se diferencian moderadamente. En términos generales, los grupos Clínico y Pericial Otros (menores víctimas) presentan las medias más altas con diferencias significativas en inadaptación general ( $\mathrm{IG}=30,88$ y IG=37,37 respectivamente; $\mathrm{p}<0,05)$. Mientras, los menores del grupo Pericial Familia apenas se diferencian de la población general en ninguna de las variables de inadaptación (Pericial Familia, IG=25,85; Población General, IG=24,22). En la figura 1 se resumen estos resultados.

En la figura 2 se puede ver un resumen de la valoración de la dinámica familiar para las distintas submuestras específicas. La insatisfacción familiar y la percepción de discrepancia educativa son significativamente mayores en el grupo Pericial Familia $(\mathrm{IF}=2,65 ; \mathrm{p}<0,01$ y $\mathrm{DE}=11 ; \mathrm{p}<0,05$ respectivamente), no encontrándose otras diferencias importantes en el resto de escalas.

Realizando un análisis ANOVA para observar el efecto del nivel de conflicto parental en las variables dependientes de adaptación y ambiente familiar, se observa que esta variable in- 


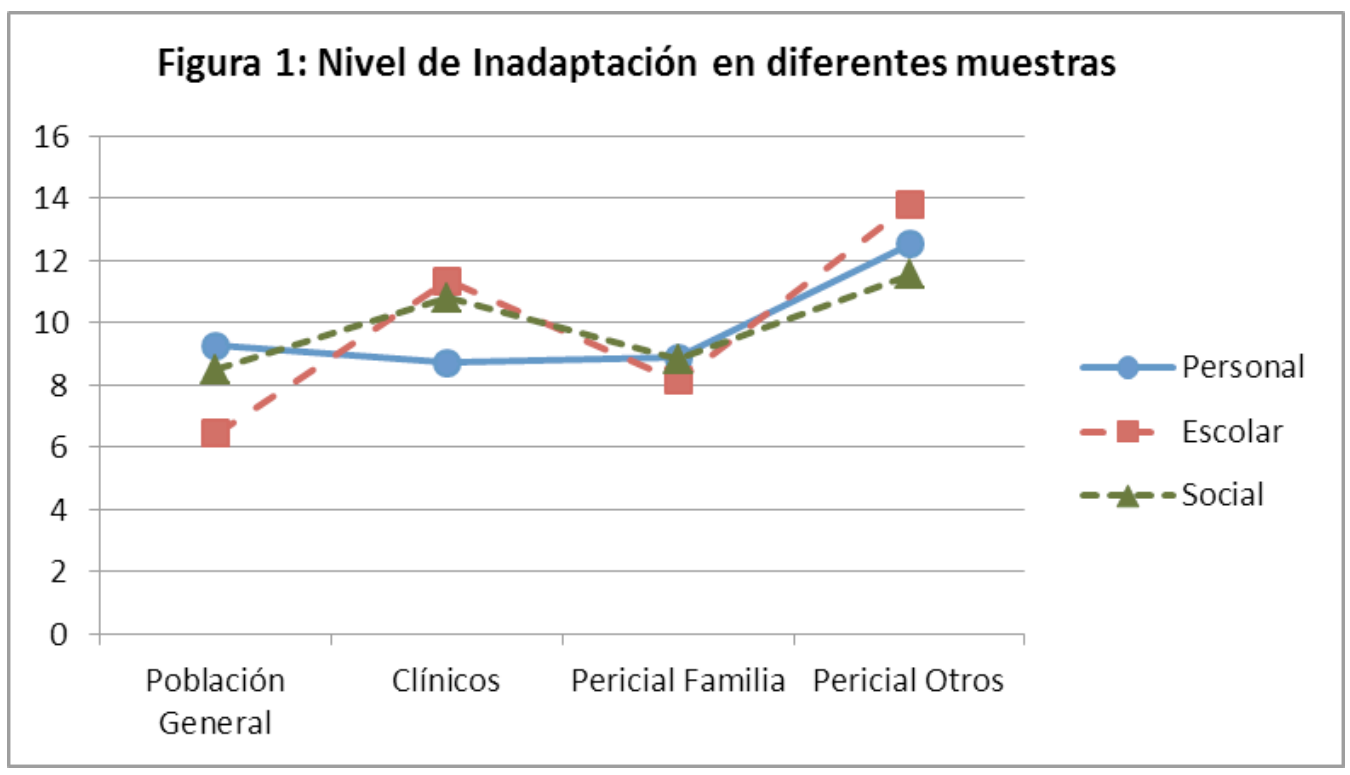

Figura 2: Ambiente familiar en grupos específicos
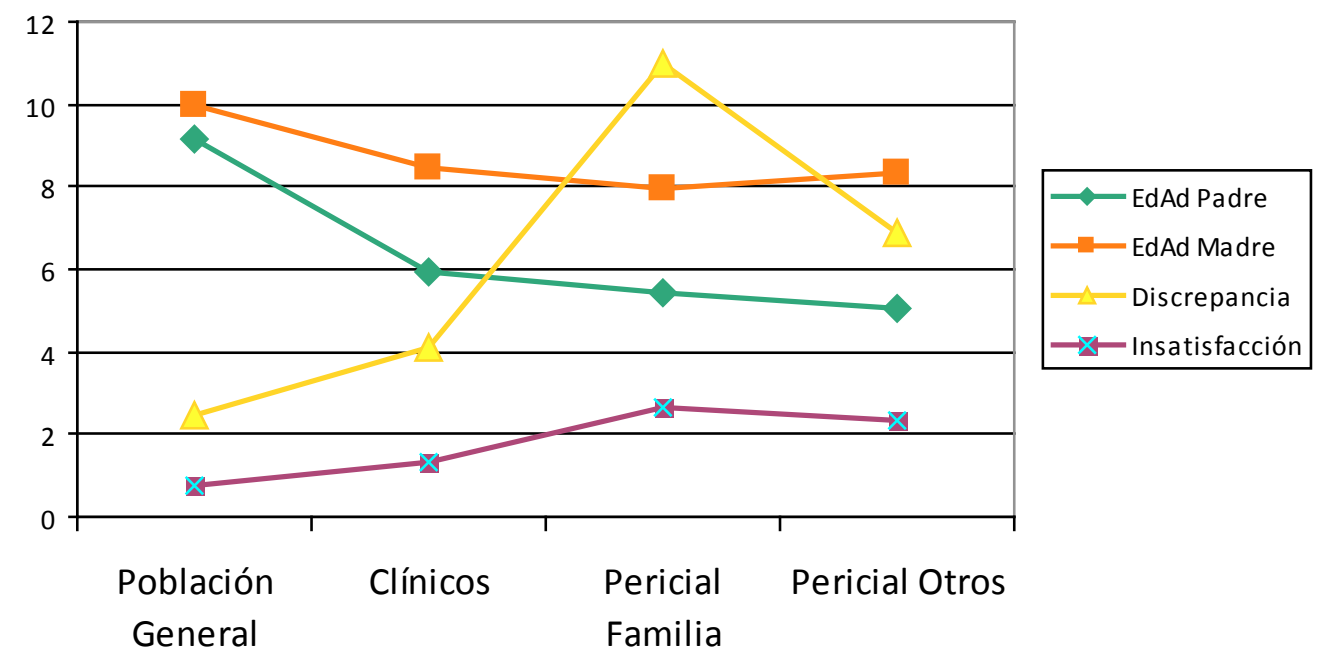
dependiente no tiene un efecto significativo sobre los niveles de inadaptación. En cambio, sí que aparecen diferencias estadísticamente significativas cuando el nivel de conflicto es alto en las variables de insatisfacción familiar $(X=2,9 ; p<0,01)$, insatisfacción con los hermanos $(X=2,19 ; p<0,05)$, Educación adecuada de la madre $(X=5,48 ; p<0,01)$ y discrepancia educativa $(X=11,24 ; p<0,01)$. Es decir, que se observa un aumento significativo de estas variables cuando los menores están inmersos en un ambiente familiar muy conflictivo, independientemente del contexto en el que hayan sido evaluados, ya que para este análisis y el siguiente se incluyeron menores de todos los grupos.

Más concretamente, se evalúa también la implicación que estos menores tienen en el conflicto familiar y se observan diferencias estadísticamente significativas indicadoras de que

\section{Tabla 3}

Anova. Efecto de la implicación en el conflicto familiar sobre variables dependientes

\begin{tabular}{|c|c|c|c|c|c|c|c|}
\hline & $\begin{array}{l}\text { Implicación } \\
\text { en conflicto }\end{array}$ & $\mathrm{N}$ & Media & $\begin{array}{l}\text { Desviación } \\
\text { típica }\end{array}$ & $\begin{array}{l}\text { Error } \\
\text { típico }\end{array}$ & $\mathrm{F}$ & Sig. \\
\hline \multirow[t]{3}{*}{ Inadaptación general } & Leve & 60 & 27.48 & 13.268 & 1.713 & \multirow{3}{*}{3.367} & \multirow{3}{*}{.039} \\
\hline & Media & 10 & 19.1 & 8.306 & 2.627 & & \\
\hline & Alta & 31 & 32.06 & 16.535 & 2.97 & & \\
\hline \multirow{3}{*}{$\begin{array}{l}\text { Inadaptación perso- } \\
\text { nal }\end{array}$} & Leve & 60 & 8.47 & 5.959 & 0.769 & \multirow{3}{*}{4.355} & \multirow{3}{*}{.015} \\
\hline & Media & 10 & 5.8 & 4.709 & 1.489 & & \\
\hline & Alta & 31 & 11.42 & 5.993 & 1.076 & & \\
\hline \multirow[t]{3}{*}{ Inadaptación escolar } & Leve & 60 & 9.42 & 6.026 & 0.778 & \multirow{3}{*}{2.919} & \multirow{3}{*}{.059} \\
\hline & Media & 10 & 4.8 & 3.458 & 1.093 & & \\
\hline & Alta & 31 & 10.42 & 7.775 & 1.396 & & \\
\hline \multirow{3}{*}{ Inadaptación social } & Leve & 60 & 9.6 & 4.673 & 0.603 & \multirow{3}{*}{.514} & \multirow{3}{*}{.600} \\
\hline & Media & 10 & 8.5 & 3.274 & 1.035 & & \\
\hline & Alta & 31 & 10.23 & 5.359 & 0.962 & & \\
\hline \multirow{3}{*}{$\begin{array}{l}\text { Insatisfacción fami- } \\
\text { liar }\end{array}$} & Leve & 60 & 1.17 & 1.498 & 0.193 & \multirow{3}{*}{16.244} & \multirow{3}{*}{.000} \\
\hline & Media & 10 & 1.6 & 1.43 & 0.452 & & \\
\hline & Alta & 31 & 3.03 & 1.472 & 0.264 & & \\
\hline \multirow{3}{*}{$\begin{array}{l}\text { Discrepancia edu- } \\
\text { cativa }\end{array}$} & Leve & 60 & 5.32 & 7.37 & 0.952 & \multirow{3}{*}{4.354} & \multirow{3}{*}{.015} \\
\hline & Media & 10 & 3.2 & 4.05 & 1.281 & & \\
\hline & Alta & 31 & 10.74 & 12.925 & 2.321 & & \\
\hline
\end{tabular}


cuanto mayor es esta implicación, mayores son los niveles de inadaptación general y personal, insatisfacción familiar y discrepancia educativa, tal y como se muestra en la tabla 3.

\section{Segunda fase}

Centrándonos en el contexto de la evaluación pericial de familia y, por tanto, en todas las implicaciones de las rupturas conyugales, en primer lugar se analizan las diferencias en las variables de inadaptación y de ambiente familiar del grupo de menores inmersos en procesos periciales (pericial civil) en comparación con la muestra comunitaria.

Con la prueba $\mathrm{T}$ de Student para muestras independientes se obtuvieron diferencias estadísticamente significativas en algunas dimensiones. En el grupo pericial civil se obtiene una mayor insatisfacción familiar $(\mathrm{t}=-6,97 ; \mathrm{p}<0,01)$, mayor discrepancia educativa $(\mathrm{t}=$ $-4,003 ; \mathrm{p}<0,01)$ y puntuaciones más bajas en el estilo educativo adecuado del padre $(\mathrm{t}=2,81$; $\mathrm{p}<0,01)$.

Así mismo, para comprobar si la situación en el ambiente familiar tiene una relación importante con los problemas en la adaptación de los niños, tanto a nivel personal como escolar y social, se realizó la prueba de correlación de Pearson obteniéndose relaciones estadísticamente significativas entre la insatisfacción familiar y la inadaptación escolar, social y personal tanto en el grupo pericial civil como en la muestra comunitaria $(\mathrm{p}<0,01)$. En el grupo pericial civil, los valores de correlación se encuentran entre $r=0,37$ y r=0,46. En el grupo de población general, los valores se encuentran entre $\mathrm{r}=0,27$ y $\mathrm{r}=0,33$.

Mediante una prueba ANOVA, se analizó el posible efecto de las variables independientes modalidad de custodia y contacto con el progenitor no custodio en la inadaptación personal, social y escolar. Los resultados muestran diferencias estadísticamente significativas únicamente en inadaptación escolar en función del régimen de convivencia previo a la evaluación $\left(\mathrm{F}_{(2,58)}=4,98 ; \mathrm{p}<0,05\right)$. La prueba de contraste a posteriori de Tukey muestra que las diferencias se encuentran entre el grupo de custodia exclusiva materna $(X=7,94)$ y el grupo de custodia exclusiva paterna $(X=17)(\mathrm{p}<0,05)$. Así mismo el coeficiente del tamaño del efecto obtenido a partir de Eta cuadrado es de 0,15 .

En cuanto a las variables independientes conflicto parental e implicación de los menores en dicho conflicto, en primer lugar se estudió su posible influencia en la insatisfacción familiar. Mediante el NOVA de un factor se obtuvieron diferencias estadísticamente significativas en insatisfacción familiar únicamente en función de la inclusión del menor en el conflicto parental $\left(\mathrm{F}_{(2,58)}=3,95 ; \mathrm{p}<0,05\right)$ tal y como se muestra en la tabla 4. Posteriormente se realizaron las pruebas de contraste a posteriori de Tukey y Dunnett y esta última mostró una diferencia estadísticamente significativa entre el grupo de inclusión baja en el conflicto $(X=1,29)$ y el grupo de inclusión alta $(X=2,57)(p<0,05)$. Por otra parte, se calculó el tamaño del efecto de la varianza mediante el coeficiente Eta cuadrado y se obtuvo un resultado de 0,12.

Además, se analizó la posible influencia de estas mismas variables (conflicto e implicación) en las dimensiones de inadaptación personal, social y escolar. El ANOVA realizado mostró que no hay diferencias estadísticamente significativas en inadaptación infantil en función del conflicto parental ni de la implicación en dicho conflicto.

La relación entre la insatisfacción familiar y la discrepancia educativa de los progenitores 


\section{Tabla 4}

Diferencias en Insatisfacción familiar en función de la inclusión del menor en el conflicto

\begin{tabular}{lccccc}
\hline & $\begin{array}{c}\text { Suma de } \\
\text { cuadrados }\end{array}$ & gl & $\begin{array}{c}\text { Media } \\
\text { cuadrática }\end{array}$ & F & Sig. \\
\hline Inter-grupos & 14.676 & 2 & 7.338 & 3.952 & .025 \\
Intra-grupos & 107.684 & 58 & 1.857 & &
\end{tabular}

percibida por los hijos obtiene un coeficiente de $\mathrm{r}=0,30(\mathrm{p}<0,05)$ calculada a partir de la correlación de Pearson. Por otra parte, la correlación entre la discrepancia educativa y las tres dimensiones de inadaptación específicas no muestra una relación estadísticamente significativa.

A partir del análisis de correlaciones de Pearson se observa una relación estadísticamente significativa entre el estilo educativo adecuado de la madre y la inadaptación personal ( $\mathrm{r}$ $=-0,30 ; \mathrm{p}<0,05)$, la inadaptación escolar $(\mathrm{r}=-0,29 ; \mathrm{p}<0,05)$ y la inadaptación social $(\mathrm{r}=$ $-0,33 ; \mathrm{p}<0,01)$. En el caso del estilo educativo adecuado del padre las relaciones no son estadísticamente significativas.

Por otro lado, los análisis realizados para observar la posible relación entre los estilos educativos, el régimen de custodia y los años transcurridos desde la ruptura conyugal con la insatisfacción familiar no muestran resultados estadísticamente significativos. En el caso del tiempo transcurrido desde la separación, tampoco se observa relación significativa con la inadaptación psicológica.

\section{Discusión}

Los menores que acuden y son atendidos en un servicio privado de psicología, bien por demanda terapéutica o bien por demanda pericial, constituyen un grupo significativamente diferente de la población general. Muestran mayor inadaptación tanto en términos generales como en términos de inadaptación escolar y social, y puntúan más alto tanto en la percepción de discrepancia entre los estilos educativos de sus progenitores como en su insatisfacción con el ambiente familiar.

La intervención psicológica por sí misma, podría indicar al menor el fracaso de los progenitores en resolver un conflicto familiar, una dolencia del menor o muestra la impotencia de los padres ante un acontecimiento traumático, por lo que no sorprende la peor valoración del entorno familiar en la muestra específica, dato que se confirma con una menor adecuación educativa de ambos progenitores para las tres submuestras, sin apreciarse diferencias significativas entre ellas, en términos generales.

Por otro lado, al comparar los tres subgrupos específicos entre ellos, observamos que los niveles de inadaptación más altos aparecen en los menores evaluados por haber sido víctimas de hechos traumáticos no relacionados con la ruptura de sus progenitores. Así mismo, los 
valores de estas variables no se diferencian entre el grupo de menores en procesos de separación conyugal y la población general. Este es un dato positivo ya que nos indica que, en términos generales, los menores que viven la separación de sus progenitores no manifiestan problemas psicológicos significativamente más graves que el resto de niños y niñas de su edad, tal y como ya se ha visto en otras investigaciones (Morgado y González, 2001).

En cambio, los menores inmersos en procesos de separación conyugal destacan por encima del resto de grupos específicos y de la muestra comunitaria en insatisfacción familiar y percepción de discrepancia educativa. Lo que nos indicaría que en general el proceso de ruptura es vívido como desagradable, afectando sobre todo a la percepción que tienen los hijos del comportamiento de sus progenitores y de los cambios que se producen en el núcleo familiar.

Además, hemos visto que la insatisfacción familiar y la discrepancia educativa están directamente relacionadas. Esto es muy importante porque las diferencias en los patrones educativos de los padres desestabilizan mucho a los menores que pueden llegar a encontrarse perdidos por la ambigüedad de las normas, de los refuerzos y castigos y de las muestras de afecto. Y cuando un niño no entiende o no sabe dónde están los límites del comportamiento adecuado e inadecuado puede establecerlos según su propio criterio o no llegar a establecerlos nunca. Éste es un factor de riesgo relevante para la inadaptación social, que aunque en nuestros resultados no se muestre directamente relacionada con la discrepancia educativa, está mediada por la insatisfacción familiar.

Por otra parte, como hemos podido comprobar las relaciones familiares influyen en todos los ámbitos de la vida de un niño/a al final de la infancia y el principio de la adolescencia, ya que todas las puntuaciones de inadaptación estaban relacionadas con la insatisfacción familiar, tanto en menores inmersos en procesos de separación conyugal como en la muestra comunitaria. Por ello, por una parte, es muy importante mantener una relación cordial y respetuosa entre los progenitores antes, durante y después del proceso de separación, porque, como han demostrado diversas investigaciones, la percepción de un ambiente de hostilidad y de venganza por parte de los menores puede alterar de forma significativa su desarrollo (Arch, 2012; Jiménez y Cano, 2014; Pons-Salvador, 2007). Y por otra, mantener una relación afectiva con los hijos, ya se trate del progenitor custodio o no custodio. Los progenitores deberían entender que los hijos no eligen la separación de sus padres y que, además, este hecho no es motivo suficiente para cambiar sus sentimientos hacia ellos. Su relación puede y debe seguir siendo la misma.

Como ya se ha dado a conocer en otros estudios, el nivel de conflicto manifiesto entre progenitores y la implicación del menor en él son de las condiciones que más influye en el malestar y en la insatisfacción de los niños (Catalán, 2015; Pons-Salvador y Del Barrio, 1995), y nuestros datos así lo apuntan.

Sin diferenciar el contexto de evaluación o de procedencia de los menores, hemos observado que un nivel elevado de conflicto familiar está relacionado con una mayor insatisfacción familiar, mayor insatisfacción con los hermanos, peor percepción del estilo educativo de la madre y mayor percepción de discrepancia educativa. Además, un nivel elevado de im- 
plicación del menor en el conflicto implica mayor inadaptación general y personal, mayor insatisfacción familiar y mayor percepción de discrepancia educativa.

Teniendo en cuenta únicamente los hijos de progenitores separados o en proceso de separación, la implicación en el conflicto familiar se ha visto relacionada significativamente con niveles más altos de insatisfacción familiar.

Por lo tanto, lo que estamos observando es que el conflicto familiar y el nivel de implicación de los menores no afectan solamente a las familias que se separan, sino que su presencia es mucho más amplia, y sus implicaciones variables en función de otros factores. En todo caso, están directamente relacionados con una mayor insatisfacción familiar y, directa o indirectamente, con mayor riesgo de inadaptación psicológica para los menores.

Otro dato que nos muestran los resultados es que el estilo educativo adecuado de la madre está relacionado de forma negativa con la inadaptación, es decir que el cuidado asistencial, la atención a las necesidades y la muestra de afecto por parte de la madre a sus hijos puede ser un factor protector a la hora de asegurar una buena adaptación personal, escolar y social de los menores.

En cambio, los resultados muestran que los hijos que conviven con sus padres tienen una peor adaptación escolar que aquellos que conviven con sus madres. No obstante, hay que tener en cuenta que únicamente el 3,3\% de los participantes evaluados dentro de un proceso judicial mantenían un régimen de convivencia exclusivo con el padre, frente al 85,25\% que mantenían un régimen de convivencia exclusivo con la madre, cosa que puede afectar tanto a estos resultados como a los reflejados en el párrafo anterior.

En resumen, podemos decir que los menores inmersos en procesos judiciales de familia se diferencian de la población general y de otros menores atendidos psicológicamente en una mayor presencia de insatisfacción familiar y de percepción de discrepancia educativa entre sus progenitores, pero no presentan mayores niveles de inadaptación psicológica en términos generales. El factor que parece tener una relación más directa con la insatisfacción familiar en esta muestra particular es la implicación en el conflicto parental. Por otra parte, la insatisfacción familiar está relacionada tanto con las tres dimensiones de inadaptación infantil (social, escolar y personal) como con la discrepancia educativa, de forma que esta implicación en el conflicto y la percepción de discrepancia educativa entre los progenitores podrían estar ejerciendo un efecto indirecto sobre la inadaptación a través de la insatisfacción familiar.

No obstante, en esta investigación debemos tener en cuenta algunas limitaciones: las diferencias en las submuestras de sujetos separados por grupos según el régimen de custodia previo y por contexto de evaluación, ya que al no tener una cantidad de sujetos similar en cada grupo, los análisis podrían no reflejar la realidad con la exactitud deseada. Pese a ello, esta distribución de niños y niñas en los diferentes grupos es representativo del contexto social en España, ya que, por una parte, las custodias exclusivas paternas siguen siendo una minoría, y por otra, las demandas de atención psicológica son mayores en el ámbito clínico que en el pericial. 
Otra limitación estaría relacionada con la utilización únicamente de un cuestionario estandarizado para evaluar la adaptación y bienestar de los menores, ya que posiblemente podría obtenerse más información, y más específica, añadiendo otras técnicas como la entrevista.

Así mismo, se han obtenido recientemente datos que apuntan hacia una carencia en el TAMAI para la detección de sintomatología de gravedad moderada, especialmente en el área de inadaptación personal. Para futuras investigaciones en este ámbito recomendamos tener en cuenta estos factores.

En conclusión, podríamos decir que a la hora de romper una relación conyugal cuando la pareja tiene hijos en común no existe una modalidad de custodia o un régimen de visitas estándar que sea más adecuado en todos los casos. Lo más adecuado será analizar cada uno en su globalidad teniendo en cuenta una gran variedad de factores, entre ellos la relación afectiva entre padres e hijos, el estilo educativo de cada progenitor, la relación entre los progenitores, el estado emocional y cognitivo de cada miembro de la familia, sus herramientas de afrontamiento de conflictos y la red social de apoyo de todo el núcleo familiar.

Además, hay que tener en cuenta dos aspectos muy importantes a la hora de minimizar el estrés y malestar en los hijos provocado por la separación, a pesar de que a priori no parece estadísticamente superior al del resto de población. Por una parte, los padres deben intentar mantener dentro de lo posible la estabilidad emocional de sus hijos demostrándoles que el cambio en la estructura familiar no debe cambiar los sentimientos y las relaciones que existen entre ellos, y por otra, deben evitar la implicación de los niños en los conflictos parentales que puedan surgir en el contexto de la ruptura. De esta forma es más probable que la adaptación de los niños a la nueva situación familiar sea más rápida y satisfactoria.

Los resultados obtenidos en este trabajo ponen de manifiesto la importancia de seguir investigando en este campo, especialmente con el objetivo de desarrollar programas específicamente diseñados para estas situaciones en los que se trabaje la comunicación, la colaboración parental o los estilos educativos con todos los agentes implicados. Al mismo tiempo, estos datos, facilitando una mejor comprensión de las situaciones familiares, pueden contribuir a desarrollar evaluaciones más precisas en el ámbito del Derecho de Familia, permitiendo así, aportar recomendaciones más específicas.

\section{Referencias}

Amato, P. (2014). The consequences of divorce for adults and children: An update. Društvena istraživanja-Časopis za opća društvena pitanja, 23(1), 5-24. doi: 10.5559/di.23.1.01

Arch, M. (2010). Divorcio conflictivo y consecuencias en los hijos: implicaciones para las recomendaciones de guarda y custodia. Papeles del Psicólogo, 31(2), 183-190.

Bauserman, R. (2002). Child adjustment in joint-custody versus sole-custody arrangements: A meta-analytic review. Journal of Family Psychology, 16(1), 91-102.

Boone-Holladay, T. (2016). Introduction to the classic edition. En A. Booth, A. C. Crouter, M. L. Clemens, y T. Boone-Holladay (Eds.), Couples in conflict. Classic edition (pp. 15-30). New York, USA: Routledge Classic Edition. 
Cantón, J., Cortés, M. R. y Justicia, M. D. (2002). Las consecuencias del divorcio en los hijos. Psicopatología Clínica, Legal y Forense, 2(3), 47-66.

Catalán, M. J. (2011). La custodia compartida. En F. De la Torre (Dir.), Revista Derecho y Criminología, Anales 2011 $n^{\circ} 1$ (pp. 57-82). Valencia, España: Tirant lo Blanch.

Catalán, M. J. (2015). Evaluación de custodias: criterios psicológicos utilizados por los Psicólogos Forenses de la Administración de Justicia (Tesis doctoral). Universidad de Murcia, Murcia, España.

Di Domenico, R. (2006). Familias separadas y apego. Revista de Psicología Segunda Época. 25(2), 6-18.

Fariña, F., Arce, R., Novo, M. y Seijo, D. (2014). La justicia terapéutica en procesos de ruptura: el papel del psicólogo. En E. C. Chan, C. Estrada y F. J. Rodríguez (Eds.), Aportaciones a la psicología jurídica y forense desde Iberoamérica (pp. 113-134). Ciudad de México, México: Manual Moderno.

Fariña, F., Martinón, J. M., Souto, A. \& García, A. (Octubre de 2014). La ruptura de los progenitores y el impacto en el estado de salud psicoemocional de los hijos. En R. Arce (Presidencia), Psicología Jurídica de la Familia. Comunicación llevada a cabo en el VIII Congreso Internacional de Psicología Jurídica y Forense, Santiago de Compostela, España.

Fariña, F., Novo, M., Arce, R. y Seijo, D. (2002). Programa de intervención "Ruptura de pareja, no de familia" con familias inmersas en procesos de separación. Psicopatología Clínica, Legal y Forense, 2(3), 67-85.

Fariña, F., Seijo, D., Arce, R. y Vázquez, M. J. (2017). Custodia compartida, corresponsabilidad parental y justicia terapéutica como nuevo paradigma. Anuario de Psicología Jurídica, 27(1), 107-113. doi: http://dx.doi.org/10.1016/j. apj.2016.11.001

Haimi, M. y Lerner, A. (2016). The impact of parental separation and divorce on the health status of children, and the ways to improve it. Journal of Clinical and Medical Genomics, 4(1), 1-7. doi: 10.4172/2472-128X.1000137

Hernández, P. (2002). TAMAl: Test Autoevaluativo Multifactorial de Adaptación Infantil. Madrid, España: TEA Ediciones.

Jiménez, P. \& Cano, M. C. (Octubre de 2014). Nivel de conflicto tras la ruptura conyugal y su efecto en los hijos. En R. Arce (Presidencia), Psicología Jurídica de la Familia. Comunicación llevada a cabo en el VIII Congreso Internacional de Psicología Jurídica y Forense, Santiago de Compostela, España.

Kelly, J. B. y Emery, R. E. (2003). Children's adjustment following divorce: risk and resilience perspectives. Family Relations: Interdisciplinary Journal of Applied Family Science, 52(4), 352-362.

Morgado, B., y González, M. M. (2001). Divorcio y ajuste psicológico infantil: primeras respuestas a algunas preguntas repetidas. Apuntes de Psicología, 19(3), 387-402.

Nielsen, L. (2014). Shared physical custody: summary of 40 studies on outcomes for children. Journal of Divorce \& Remarriage, 55(8), 613-635. doi: 10.1080/10502556.2014.965578

Novo, M., García, A. y Carracedo, S. (Octubre de 2014). El impacto de la ruptura de los progenitores en el ámbito académico y escolar de los ninos. En R. Arce (Presidencia), Psicología Jurídica de la Familia. Comunicación llevada a cabo en el VIII Congreso Internacional de Psicología Jurídica y Forense, Santiago de Compostela, España.

Pons-Salvador, G. y Del Barrio, V. (1995). El efecto del divorcio sobre la ansiedad de los hijos. Psicothema, 7(3), 489-497.

Pons-Salvador, G. (2007). La influencia del divorcio sobre los hijos: factores moduladores del impacto psicológico. Letras de Deusto, 37(115), 109-121.

Ríos Sarrió, A. (2009). Perspectivas psicológicas de los conflictos familiares: la familia y el divorcio. En J. Piñero, F. Vargas y E. Torres. (Coords.), Puntos de encuentro familiar: manual de uso práctico, (pp. 61-80). Elche, España: Fundación Salud Infantil.

Seijo, D., Novo, M., Carracedo, S. y Fariña, F. (2010). Efectos de la ruptura de los progenitores en la salud física y psicoemocional de los hijos. Revista Galega de Cooperación Científica Iberoamericana, 19, 16-21.

Tejedor, M. A. (2012). El interés de los menores en los procesos contenciosos de separación o divorcio. Anuario de Psicología Jurídica, 22(1), 67-75. doi: http://dx.doi.org/10.5093/aj2012a7 